\title{
Molecular characterization of virulence genes of Streptococcus equi subsp. equi and Streptococcus equi subsp. zooepidemicus in equines
}

\author{
R. Javed ${ }^{1}$, A. K. Taku ${ }^{1}$, Rakhi Gangil ${ }^{2}$ and R. K. Sharma
}

1. Department of Microbiology, Faculty of Veterinary Sciences \& Animal Husbandry, R.S. Pura, Jammu, Jammu and Kashmir, India; 2. Department of Microbiology, College of Veterinary Science and Animal Husbandry, Mhow, Madhya Pradesh, India.

Corresponding author: Rakhi Gangil, e-mail: rgangil@gmail.com,

RJ : rabiajavedkhan@gmail.com, AKT: aniltaku@gmail.com, RKS: rajni.psc@gmail.com

Received: 04-06-2016, Accepted: 16-07-2016, Published online: 19-08-2016

doi: 10.14202/vetworld.2016.875-881 How to cite this article: Javed R, Taku AK, Gangil R, Sharma RK (2016) Molecular characterization of virulence genes of Streptococcus equi subsp. equi and Streptococcus equi subsp. zooepidemicus in equines, Veterinary World, 9(8): 875-881.

\begin{abstract}
Aim: The aim was to determine the occurrence of streptococci in equines in Jammu (R. S. Pura, Katra), characterization of Streptococci equi subsp. equi and Streptococcus equi subsp. zooepidemicus with respect to their virulence traits and to determine antibiotic sensitivity pattern of virulent Streptococcus isolates.

Materials and Methods: A total of 96 samples were collected from both clinically affected animals (exhibiting signs of respiratory tract disease) and apparently healthy animals and were sent to laboratory. The organisms were isolated on Columbia nalidixic acid agar containing 5\% sheep blood as well as on sheep blood agar and confirmed by cultural characteristics and biochemical tests. Molecular detection of Streptococcus was done directly from cultures using sodA and seM gene-based polymerase chain reaction (PCR). Antibiogram was performed against five antibiotics such as amoxicillin, penicillin $\mathrm{G}$, streptomycin, rifampicin, and methicillin.

Results: During this study, a total 40 streptococcal isolates were obtained out of which 2 isolates were of $S$. equi subsp. equi, 12 isolates were from S. equi subsp. zooepidemicus. In the PCR-based detection, we revealed amplicons of 235 bp and $679 \mathrm{bp}$ for confirmation of sodA and seM gene, respectively. In antibiogram, two isolates of $S$. equi subsp. equi were found resistant to penicillin $\mathrm{G}$, and all other isolates were found sensitive to amoxicillin and streptomycin.
\end{abstract}

Conclusion: The majority of streptococcal infections was due to S. equi subsp. Zooepidemicus, and thus was recognized as a potential pathogen of diseases of equines besides $S$. equi subsp. equi.

Keywords: polymerase chain reaction, Streptococcus equi sub sp. equi, and Streptococcus equi sub sp. zooepidemicus.

\section{Introduction}

Equines play an important role in the socio-economic life of human population. They have been playing an important role in tourism promotion. Equines are used for working purposes, sports, and leisure activities, and nowadays, as means of transportation in the tourism industry. In Jammu and Kashmir, equine population constitutes the most preferred and economic means of transport used by tourists to visit various high altitude places including various places of religious importance. The total equine population in India is approximately 5.3 million, out of which 2.39 million (13.9\%) is present in Jammu and Kashmir which stands at second position [1]. Upper respiratory tract infections are common in horses and can be caused by viral, fungal, and bacterial pathogens. Although a variety of bacterial agents have been associated with the respiratory problems in equines,

Copyright: J aved, et al. Open Access. This article is distributed under the terms of the Creative Commons Attribution 4.0 International License (http://creativecommons.org/licenses/by/4.0/), which permits unrestricted use, distribution, and reproduction in any medium, provided you give appropriate credit to the origina author(s) and the source, provide a link to the Creative Commons license, and indicate if changes were made. The Creative Commons Public Domain Dedication waiver (http://creativecommons.org/ publicdomain/zero/1.0/) applies to the data made available in this article, unless otherwise stated the most important ones which are associated with upper respiratory tract diseases include streptococci (Streptococcus equi subsp. equi and S. equi subsp. zooepidemicus) and Rhodococcus equi [2]. Bacteria belonging to the genus Streptococcus are Grampositive cocci that form chains or occur in pairs. Streptococci can be host specific or be transmitted between, and cause disease in, several species including zoonotic transmission to humans [3,4]. Molecular typing methods showed that human and equine isolates of Streptococcus zooepidemicus were identical or closely related; it emphasize that transmitted from horses can lead to severe infections in humans [5].

The most commonly isolated beta-hemolytic streptococci from horses with respiratory and genital diseases are $S$. equi subsp. equi and $S$. equi subsp. zooepidemicus [4]. S. equi subsp. equi is the most notorious agent associated with great economic losses to equine husbandry by affecting the pulmonary infections and reducing their performance. S. equi subsp. zooepidemicus is the ancestor of $S$. equi and is generally considered an opportunistic commensal of the equine upper respiratory tract $[6,7]$. S. equi spp. zooepidemicus is associated with a wide variety of infections in many animal species including horses, cows, swine, sheep, and dogs [8,9]. S. equi 
spp. zooepidemicus is most frequently isolated from the cases of equine pneumonia and pleuropneumonia [10], but it is also associated with infectious endometritis in the mare [11]. Upper respiratory disease caused by $S$. zooepidemicus can mimic mild cases of strangles [12], and the subspecies can also be isolated from horses with confirmed $S$. equi infection [13].

Most of the respiratory diseases are contagious; therefore, there is a great demand by clinicians and horse owners for earlier laboratory confirmation. Unfortunately, very less research has been done in India despite the high population in equines and their importance. Keeping these facts in view, this study was envisaged with the objective of determination of the prevalence of different species streptococci (S. equi subsp. zooepidemicus and S. equi subsp. equi) and characterization of their virulence factors and antibiotic sensitivity patterns.

\section{Materials and Methods}

\section{Ethical approval}

The approval from the Institutional Animal Ethics Committee (IAHC) to carry out this study was not required as no invasive technique was used. Nasal swab samples were being collected from clinically affected animals and healthy animals for this study as per standard collection procedure.

\section{Sample collection}

The present investigation was conducted during August 2013 to July 2014. Samples were collected from R. S. Pura and Katra regions of Jammu (Jammu and Kashmir). A total of 96 nasal swab samples were collected aseptically from 50 clinically affected animals (exhibiting signs of respiratory tract disease) and 46 apparently healthy animals up to 6 months age. The samples were immediately transported to the laboratory of Department of Veterinary Microbiology and Immunology, Faculty of Veterinary Sciences and Animal Husbandry, Sher-e-Kashmir University of Agricultural Sciences and Technology of Jammu, R. S. Pura for further processing.

\section{Isolation and biochemical characterization of bacteria}

Nasal swab samples were inoculated in Todd Hewitt broth and brain heart infusion (BHI) broth for enrichment at $37^{\circ} \mathrm{C}$ for $4 \mathrm{~h}$. From these enrichment broths, the samples were inoculated on blood agar plates (containing 5\% sheep blood) and Columbia nalidixic agar plates and incubated at $37^{\circ} \mathrm{C}$ for $48 \mathrm{~h}$ for the isolation of streptococci. The bacterial isolates which showed smooth translucent, shiny colonies with zones of $\beta$-hemolysis were selected for further processing. Pure colonies of bacteria were obtained by sub-culturing (2-3 times) on the 5\% sheep blood agar. The bacterial isolates were presumptively identified based on color and status of hemolysis and Gram's staining. All the Streptococcal isolates obtained were subjected to biochemical characterization using Histrep Identification Kit (KB 005A, Himedia,
Mumbai, India). The kit contains 12 biochemical tests which include Voges-Proskauer, esculin hydrolysis, pyrrolidonyl arylamidase test, O-nitrophenyl- $\beta$-dgalactopyranoside, arginine utilization, and fermentation of seven sugars, viz., glucose, lactose, arabinose, sucrose, sorbitol, mannitol, and raffinose.

\section{Molecular detection of Streptococcus by polymerase chain reaction (PCR)}

Species-specific PCR was used for detection of important bacterial pathogens from bacterial isolates targeting 16SrRNA genus-specific PCR for Streptococcus, SeM gene for $S$. equi subsp. equi (S. equi subsp. equi), sodA gene for $S$. equi subsp. zooepidemicus (S. equi subsp. zooepidemicus), and $16 S$ rRNA. SeM gene encodes for the M like protein exclusively found in $S$. equi subsp. equi, which protects it from phagocytosis and renders it highly pathogenic while sodA gene encodes for superoxide dismutase enzyme which neutralizes the effect of superoxides by the host thereby protecting it from getting killed by neutrophils. SeM gene was amplified by PCR with light modification in given protocol [14]. PCR was carried out in a final reaction volume of $25 \mu \mathrm{l}$ using $0.2 \mathrm{ml}$ thin wall sterile and nuclease free PCR tubes. The PCR mixture contained a final concentration of $1.5 \mathrm{mM} \mathrm{MgCl}, 0.20 \mathrm{mM}$ concentrations of each 2'-deoxynucleoside 5'-triphosphate (dNTPs), $2.5 \mu 1$ of $\times 10$ PCR buffer, $1.0 \mu \mathrm{M}$ of forward and reverse primers, 1.0 U of Taq DNA polymerase (Promega limited U.S.A), and 3.0 $\mu$ l template DNA. The primers used in this study were procured from Chromous Biotech Pvt. Ltd., Bengaluru, India. The oligonucleotide primers used in the present study and predicted size of the PCR amplicon according to the previous study (Table-1) [15].

Amplification was carried out in a thermal cycler (Eppendorf Mastercycler Gradient, Germany). The amplification cycle consisted of initial denaturation at $94^{\circ} \mathrm{C}$ for $2 \mathrm{~min}$, followed by 35 cycles, each consisting of initial denaturation at $94^{\circ} \mathrm{C}$ for $10 \mathrm{~s}$, annealing at $56^{\circ} \mathrm{C}$ for $10 \mathrm{~s}$ and extension at $72^{\circ} \mathrm{C}$ for $5 \mathrm{~s}$ which was followed by final extension at $72^{\circ} \mathrm{C}$ for $5 \mathrm{~min}$. sodA gene was amplified by PCR with slight modifications [16]. PCR was carried out in a final reaction volume of $25 \mu \mathrm{l}$ in $0.2 \mathrm{ml}$ thin wall sterile and nuclease free PCR tubes (Eppendorf, Germany). The PCR mixture contained a final concentration of $1.5 \mathrm{mM} \mathrm{MgCl}_{2}$, $0.20 \mathrm{mM}$ concentrations of each dNTPs, $2.5 \mu 1$ of $\times 10$ PCR buffer, $1.0 \mu \mathrm{M}$ of forward and reverse primers,

Table-1: List of oligonucleotide primers for detection of SeM gene (Timoney and Artiuschin, 1997).

\begin{tabular}{lcc}
\hline Primer name Nucleotide sequence & $\begin{array}{c}\text { Product } \\
\text { size } \\
\text { (bp) }\end{array}$ \\
\hline $\begin{array}{l}\text { Forward primer 5' TGCATAAAGAAGTTCCTGTC 3' } \\
\text { (SeM 7) }\end{array}$ & 679 \\
Reverse primer 5' GATTCGGTAAGAGCTTGACG 3' \\
(SeM 6)
\end{tabular}


$3.00 \mu 1$ template DNA and $1.0 \mathrm{U}$ of Taq DNA polymerase (Promega Limited, USA). Primer sequences used in the study and predicted size of the PCR amplicon according to the previous study (Table-2) [16]. Amplification was carried out in a thermal cycler (Eppendorf Mastercycler Gradient, Germany) in the similar manner as that for $S$. equi subsp. equi. The confirmation of PCR product was done by electrophoresis of amplified products in $1.0 \%$ agarose gel in horizontal electrophoresis unit (Biometra, Germany). This gel was visualized under BioDocAnalyze (Biometra) and photographed. Molecular sizes at of standard molecular size marker.

\section{Antimicrobial sensitivity assay}

All the isolates of S. equi subsp. equi, S. zooepidemicus, and $R$. equi were subjected to antibiotic sensitivity test by disc diffusion method [17] using five antimicrobials, amoxiciin $-30 \mu \mathrm{g}$, penicillin $\mathrm{G}-25 \mathrm{U}$, amikacin - $30 \mu \mathrm{g}$, streptomycin - $10 \mu \mathrm{g}$, and methicillin - $5 \mu \mathrm{g}$ (Himedia, Mumbai, India). The antibiotic sensitivity assay was performed on 5\% sheep blood agar. Two to three bacterial colonies were picked from culture plate and inoculated in the BHI and incubated at $37^{\circ} \mathrm{C}$ for $6 \mathrm{~h}$. A sterile cotton swab was dipped in the BHI, and swab was inoculated by lawn method on $5 \%$ sheep blood agar. The test antibiotic discs were dispensed by antibiotic disc dispenser (Himedia, Mumbai, India).

\section{Results}

In this study, out of 96 samples ( 50 from diseased and 46 apparently healthy), total 218 bacterial isolates were obtained. From these bacterial isolates, 121 recovered from diseased animal and 97 from healthy animals. The majority of bacteria isolated (97 out of 121) from diseased animals were Gram-positive while others were Gram-negative. Similarly, 63 isolates from apparently healthy animals were Gram-positive while 34 were Gram-negative. Streptococci were presumptively identified as small, smooth, shiny, and hemolytic colonies $(\alpha / \beta)$ on $5 \%$ sheep blood agar and were purified by repeated sub-culturing on $5 \%$ sheep blood agar (Figure-1). Gram's staining of these isolates revealed Gram-positive cocci in chain (Figure-2). 40 isolates of streptococci were identified based on cultural characteristics and Gram-staining was subjected to a series of biochemical tests for further characterization of the isolates. All the 40 streptococcal isolates were subjected to genus-specific $16 \mathrm{~S}$ rRNA PCR for confirmation as streptococci. Out of 40 isolates, 20 were confirmed as belonging to genus Streptococcus. Out of these 20 isolates, 16 belonged to diseased animals while four were from apparently healthy animals. Confirmed streptococcal isolates were further subjected to species-specific PCR-based on virulence genes.

All the 20 streptococcal isolates confirmed by 16S rRNA genus-specific primers were subjected to SeM PCR for detection of $S$. equi subsp. equi, and $2(4.25 \%)$ an amplicon of 679 bp confirmatory for S. equi subsp. equi and other isolates were subjected to PCR for detection of sodA gene of S. equi subsp. zooepidemicus which revealed an amplicon of 235 bp confirmatory for S. equi subsp. zooepidemicus (Figure-3). PCR based results for $S$. equi subsp. equi and $S$. equi subsp. zooepidemicus from apparently healthy and diseased equines is given in Tables- 3 and 4.

The summary of the results of antibiotic sensitivity of two isolates of S. equi ssp. equi are shown in Table-5. The results revealed that amoxicillin and rifampicin were the most effective followed by streptomycin while maximum resistance was noted against penicillin G. Both the streptococcal isolates demonstrated intermediate

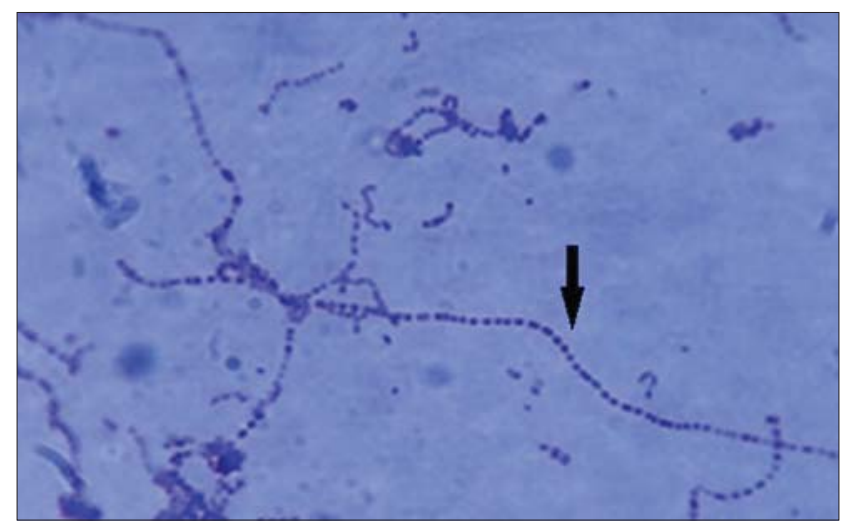

Figure-1: Long chains of Streptococcus equi subsp. equi Gram-staining.

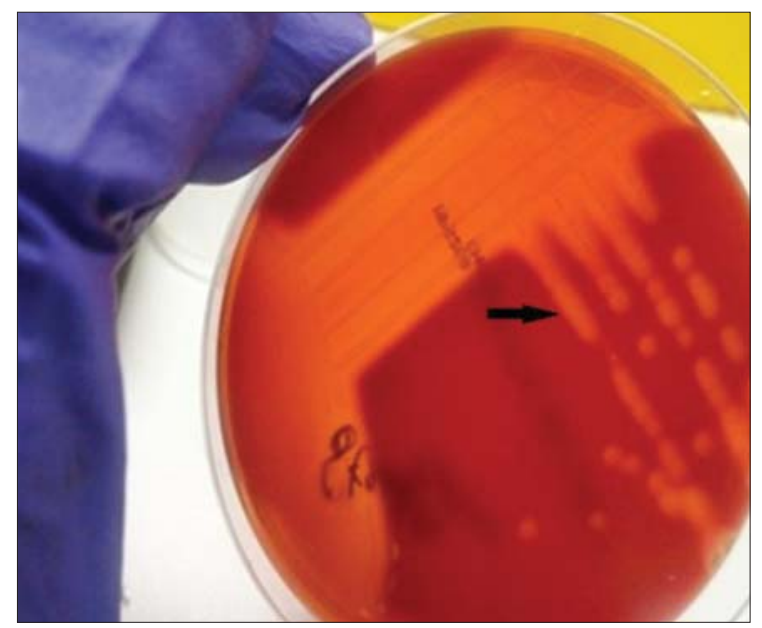

Figure-2: Streptococcus equi showing beta hemolysis on blood agar.

Table-2: List of oligonucleotide primers for detection of sodA gene (Alber et al., 2004).

\begin{tabular}{llc}
\hline Primer name & Nucleotide sequence & Product size (bp) \\
\hline Forward primer & 5'-CAGCATTCCTGCTGACATTCGTCAGG 3' & 235 \\
Reverse primer & 5'-CTGACCAGCATTATTCACAACCAGCC 3' & \\
\hline
\end{tabular}


zones with methicillin. The results of antibiotic sensitivity assay of 12 isolates of $S$. equi ssp. zooepidemicus are presented in Table-5. The results revealed that amoxicillin and rifampicin were the most effective for S. equi subsp. zooepidemicus followed by streptomycin. The results further revealed that resistance was highest for penicillin $\mathrm{G}$ followed by methicillin.

\section{Discussion}

Although a variety of bacterial agents have been associated with the respiratory problems in equines,

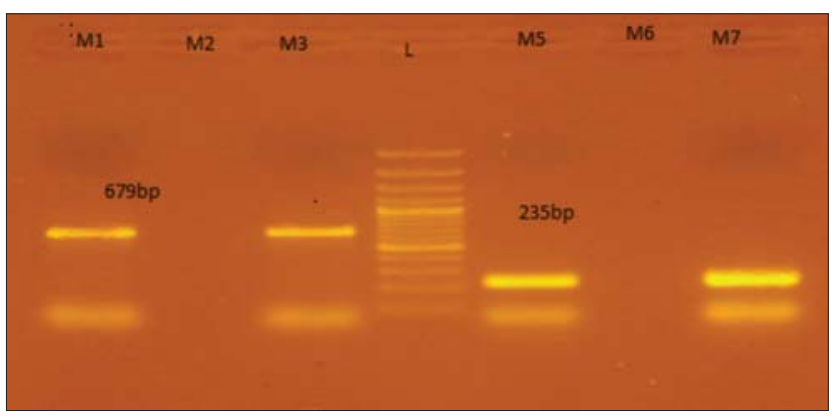

Figure-3: Amplified product of Streptococcus equi and Streptococcus zooepidemicus. Lane M1, M3: Amplified product of S. equi for SeM gene at $679 \mathrm{bp}$, Lane M2, M6: Negative sample, Lane M5, M7: Amplified product of S. zooepidemicus for sodA gene at $235 \mathrm{bp}$, Lane L: Molecular weight marker of $100 \mathrm{bp}$, (Himedia India). the most important ones which are associated with upper respiratory tract diseases include streptococci (S. equi subsp. equi and S. equi subsp. zooepidemicus) [18]. During our study, isolation of streptococci remained standard for the diagnosis of these bacteria but due to the fastidious nature and time involved in the diagnosis, by routine culturing, PCR was attempted for detection of these bacteria directly from the pure isolates. Moreover, PCR assay is more rapid and sensitive [14] than microbiologic culture and is highly specific [19].

In this study, a total of 218 isolates were recovered from 96 samples. When the information generated was compared to the health status of animals, it was found that isolation rate was 121 isolates from 50 samples of diseased animals compared to 97 isolates from 46 samples of apparently healthy animals. Ijaz et al. also reported higher prevalence of $S$. equi subsp. equi during early spring season only [20]. However, Malik and Kalra did not find any relation of the availability of cases of strangles or other respiratory tract diseases with the season [21]. This may be due to the fact that in their study they selected mostly tropical areas where the effect of temperature variation was not significant. Recently, an outbreak of strangles in 200 horses due S. equi subsp. equi was

Table-3: Distribution of S. equi subsp. equi as detected by amplification of SeM gene from apparently healthy and diseased equines.

\begin{tabular}{|c|c|c|c|c|c|c|}
\hline \multirow[t]{2}{*}{ Region } & \multicolumn{2}{|c|}{ Diseased animals } & \multicolumn{2}{|c|}{ Apparently healthy animals } & \multicolumn{2}{|l|}{ Total } \\
\hline & $\begin{array}{c}\text { Number of isolates } \\
\text { as detected from } \\
\text { 16SrRNA }\end{array}$ & $\begin{array}{l}\text { Positive for } \\
\text { SeM gene }\end{array}$ & $\begin{array}{c}\text { Number of isolates } \\
\text { as detected from } \\
\text { 16SrRNA }\end{array}$ & $\begin{array}{l}\text { Positive for } \\
\text { SeM gene }\end{array}$ & $\begin{array}{c}\text { Number of isolates } \\
\text { as detected from } \\
\text { 16SrRNA }\end{array}$ & $\begin{array}{c}\text { Positive for } \\
\text { SeM gene }\end{array}$ \\
\hline R.S. Pura & 4 & 0 & 1 & 0 & 5 & 0 \\
\hline Katra & 12 & 2 & 3 & 0 & 15 & 2 \\
\hline Total & 16 & 2 & 4 & 0 & 20 & 2 \\
\hline
\end{tabular}

S. equi=Streptococcus equi

Table-4: PCR-based distribution of sodA gene from apparently healthy and diseased equines.

\begin{tabular}{|c|c|c|c|c|c|c|}
\hline \multirow[t]{2}{*}{ Region } & \multicolumn{2}{|c|}{ Diseased } & \multicolumn{2}{|c|}{ Apparently healthy } & \multicolumn{2}{|l|}{ Total } \\
\hline & $\begin{array}{c}\text { I solates subjected } \\
\text { to PCR }\end{array}$ & $\begin{array}{l}\text { Positive for } \\
\text { sodA gene }\end{array}$ & $\begin{array}{c}\text { I solates subjected } \\
\text { to PCR }\end{array}$ & $\begin{array}{l}\text { Positive for } \\
\text { sodA gene }\end{array}$ & $\begin{array}{c}\text { I solates subjected } \\
\text { to PCR }\end{array}$ & $\begin{array}{l}\text { Positive for } \\
\text { sodA gene }\end{array}$ \\
\hline R. S. Pura & 4 & 2 & 1 & 1 & 5 & 3 \\
\hline Katra & 10 & 8 & 3 & 1 & 13 & 9 \\
\hline Total & 14 & 10 & 4 & 2 & 18 & 12 \\
\hline
\end{tabular}

$\mathrm{PCR}=$ Polymerase chain reaction

Table-5: Results of antibiogram for S. equi subsp. equi and S. equi subsp. zooepidemicus.

\begin{tabular}{|c|c|c|c|c|c|c|}
\hline \multirow[t]{2}{*}{ Antibiotics } & \multicolumn{3}{|c|}{ S. equi subsp. equi ( $N=2)$} & \multicolumn{3}{|c|}{ S. equi subsp. zooepidemicus $(\mathrm{N}=12)$} \\
\hline & $\begin{array}{c}\text { Sensitive } \\
\text { isolates (\%) }\end{array}$ & $\begin{array}{c}\text { Resistant } \\
\text { isolates (\%) }\end{array}$ & $\begin{array}{l}\text { Intermediate } \\
\text { isolates (\%) }\end{array}$ & $\begin{array}{c}\text { Sensitive } \\
\text { isolates (\% ) }\end{array}$ & $\begin{array}{c}\text { Resistant } \\
\text { isolates (\%) }\end{array}$ & $\begin{array}{l}\text { Intermediate } \\
\text { isolates (\%) }\end{array}$ \\
\hline Amoxicillin & 100 & 0 & 0 & 83.33 & 16.66 & 0 \\
\hline Penicillin G & 0 & 50 & 50 & 16.66 & 75 & 8.3 \\
\hline Streptomycin & 50 & 0 & 50 & 66.66 & 16.66 & 16.66 \\
\hline Rifampicin & 100 & 0 & 0 & 75 & 25 & 0 \\
\hline Methicillin & 0 & 0 & 100 & 0 & 58.33 & 41.66 \\
\hline
\end{tabular}

S. equi=Streptococcus equi 
reported in the UK that led to significant economic and welfare costs [22].

An outbreak of strangles was reported in the horses working at brick kilns in Jammu, India [23]. They found 38 out of 43 animals to be infected and recorded morbidity of $88.37 \%$. The diagnosis was made by them on the basis of clinical symptoms and microbiological culture of the nasopharyngeal swabs.

Application of the PCR technique was previously described for the detection of $S$. equi subsp. equi in nasal and abscess swabs from the horses in a local stud farm and a quarantine station in Malaysia [24]. They used conventional culture method and SeM based PCR for the detection of $S$. equi subsp. equi. They were unable to detect the $S$. equi subsp. equi from any of the samples. The higher prevalence and diversity of SeM gene was also reported in strangles outbreak in Brazil [25].

Out of 18 samples were subjected to PCR for detection of $S$. equi subsp. zooepidemicus were taken directly from pure isolated colonies, out of which 12 were found to be positive for sodA gene. The high recovery rate of this bacterium from the upper respiratory tract of equines has also been reported by Malik and Kalra who obtained 16 isolates of $S$. equi subsp. zooepidemicus from a total of 35 isolates of streptococci recovered in their study [21]. Similar findings had been reported by Jannatabadi et al. who got 25 isolates of S. equi subsp. zooepidemicus from 30 cases of respiratory diseases of equines [26]. In contrary to my findings in a recent study where S. zooepidemicus was isolated from tracheal washes in only $21 \%$ of healthy horses [27].

Thus, it seems from frequent recovery of $S$. equi subsp. zooepidemicus from cases of respiratory diseases that it is involved in causing mild respiratory diseases [12] besides S. equi subsp. equi. Although the role of S. equi subsp. zooepidemicus as primary bacterial pathogen remains debatable, the most recognize it as the most common bacterial pathogen isolated from equine cases [18]. Mir et al. conducted bacteriological and molecular detection of $S$. equi sub sp. equi and S. equi subsp. zooepidemicus in equines of Northern India [28].

A total of 141 samples were collected in duplicate from nasopharyngeal tract of diseased (53) and apparently healthy equines (88) for isolation and direct PCR reported PCR more sensitive than that of routine laboratory culture technique [12]. The reason for less recovery through culture technique could be less number of bacteria present in the mucosal epithelium due to the efficient mucociliary apparatus of equines and rapid desiccation of the bacteria outside the host. Another possible reason responsible for obtaining less number of isolates could be failure of transfer of organism from swab sample to the culture plates. Since the diagnosis of Streptococcus zooepidemicus, on the basis of the isolation of S. equi subsp. zooepidemicus is difficult and time consuming process, not only because of the slow-growing and fastidious nature of this facultative anaerobe, but also because of the overgrowth of a large number of different bacteria. Therefore, we suggest that sodA based PCR assay should be used for rapid identification of S. equi subsp. zooepidemicus. Recently, sodA gene characterized by real-time PCR in Uppasala [29].

Growth of $S$. equi grown on sheep blood agar and Columbia nalidixic agar plate was very slow and very difficult to visualize initially, due to the small size of the colonies. However, on confirmation by Gram-staining and PCR analysis, S. equi subsp. equi, and S. equi subsp. zooepidemicus were found to be present in the cultures. Thus, the presence of $S$. equi subsp. equi and $S$. equi subsp. zooepidemicus in the cultures were in agreement with other findings of who reported that Columbia nalidixic agar media improves the growth and facilitates the isolation of the organism [30]. The probable reason can be attributed to the sporadic nature of the disease [31]. The importance of $S$. equi subsp. zooepidemicus as a cause of upper respiratory disease with the potential to be transmitted between horses and cause outbreaks is currently being investigated [32].

To find the of drug resistance of bacteria, all the $S$. equi isolates were sensitive to amoxicillin and streptomycin. Most of the isolates were resistant to penicillin $\mathrm{G}$ and methicillin. Most of the S. equi subsp. zooepidemicus isolates were sensitive to amoxicillin and Streptomycin and resistant to penicillin G streptococcal isolations. Earlier reports of the antimicrobial sensitivity on streptococcal isolates also indicated a wide variation in the sensitivity to various antibiotics used [33,34]. Resistance of the some of the isolates to a number of antibiotics seems to be outcome of indiscriminate use of those antibiotics in the field. The antibiogram study indicates that amoxicillin and streptomycin are the effective drugs used against bacterial pathogens. However, the emergence of drug resistance bacteria can be alarming which needs close and repeated vigilance. The indiscriminate use of antibiotics should be avoided. The different combination of antibiotics should be used from time to time.

\section{Conclusion}

From this study, it was concluded that the majority of streptococcal infections were due to $S$. equi subsp. Zooepidemicus, and thus was recognized as a potential pathogen of diseases of equines besides $S$. equi subsp. equi. These pathogens were detected directly by SeM, sodA, and 16SrRNA (species specific) gene amplification. Furthermore, it was found that indiscriminate use of the antibiotics is leading toward the development of resistant strains of Streptococcus.

\section{Authors' Contributions}

RJ and AKT designed the study. Laboratory work was done by RJ and AKT. RJ, AKT, RG and RKS all the authors participated in data analysis, 
while RJK and RG have drafted and revised the manuscript. All authors read and approved the final manuscript.

\section{Acknowledgments}

The authors are thankful to Dean of Faculty of Veterinary Sciences and Animal Husbandry and Animal House, R.S. Pura, Jammu, for providing necessary research facilities. The authors also thankful to Indian Council of Medical Research (ICMR) for providing funds for the research work (ICMR grant - 30/3/18/2008/ECD/II).

\section{Competing I nterests} interests.

The authors declare that they have no competing

\section{References}

1. Anonymous, Government of India. (2008) All India Summary Report, $18^{\text {th }}$ Indian Livestock Census, Ministry of Agriculture.

2. Takai, S. (1997) Epidemiology of Rhodococcus equi infections: A review. Vet. Microbiol., 56: 167-176.

3. Fulde, M. and Valentin-Weigand, P. (2013) Epidemiology and pathogenicity of zoonotic streptococci. Curr. Top. Microbiol., 368: 49-81.

4. Quinn, P.J., Markey, B.K., Leonard, F.C., FitzPatrick, E.S., Fanning, S. and Hartigan, P.J. (2011) Veterinary Microbiology and Microbial Disease. $2^{\text {nd }}$ ed. Blackwell Science Ltd., Chichester, West Sussex, UK

5. Pelkonen, S., Lindahl, B.S., Suomala, P., Karhukorpi, J., Vuorinen, S., Irma, K., Väisänen, T., Pentikäinen, J., Autio, T. and Tuuminen, T. (2013) Transmission of Streptococcus equi subspecies zooepidemicus infection from horses to humans emerging infectious diseases. 19: 7. Available from: http:// www.cdc.gov/eid. Accessed on 09-07-2016.

6. Anzai, T., Walker, J., Blair, M.B., Chambers, T.M. and Timoney, J.F. (2000) Comparison of the phenotypes of Streptococcus zooepidemicus isolated from the tonsils of healthy horses and specimens obtained from foals and donkeys with pneumonia. Am. J. Vet. Res., 61: 162-166.

7. Waller, A.S., Paillot, R. and Timoney, J.F. (2011) Streptococcus equi: A pathogen restricted to one host. J. Med. Microbiol., 60: 1231-1240.

8. Acke, E., Midwinter, A.C., Lawrence, K., Gordon, S.J., Moore, S., Rasiah, I., Steward, K., French, N. and Waller, A. (2015) Prevalence of Streptococcus dysgalactiae subsp. equisimilis and S. equi subsp. zooepidemicus in a sample of healthy dogs, cats and horses. NZ. Vet. J., 63: 265-271.

9. Bisgaard, M., Bojesen, A.M., Petersen, M.R. and Christensen, H. (2012) A major outbreak of Streptococcus equi subsp. zooepidemicus infections in free-range chickens is linked to horses. Avian Dis., 56(3): 561-566.

10. Sweeny, C.R., Holocombe, S.J., Barningham, S.C. and Beech, J.M. (1991) Aerobic and anaerobic bacterial isolates from horse with pneumonia on pleuropneumonia and antimicrobial sensitivity patterns of the isolates. J. Am. Vet. Med. Assoc., 198: 839-842.

11. Rasmussen, C.D., Haugaard, M.M., Petersen, M.R., Nielsen, J.M., Pedersen, H.G. and Bojesen, A.M. (2013) Streptococcus equi subsp. zooepidemicus isolates from equine infectious endometritis belong to a distinct genetic group. Vet. Res., 44: 26.

12. Laus, F., Preziuso, S., Spaterna, A. and Beribe, F. (2007) Clinical and epidemiological investigation of chronic upper respiratory diseases caused by beta haemolytic streptococci in horses. Comp. Immunol. Microbiol., 12: 247-260.

13. Webb, K., Barker, C., Harrison, T., Heather, Z.,
Steward, K.F., Robinson, C., Newton, J.R. and Waller, A.S. (2013) Detection of Streptococcus equi subspecies equi using a triplex qPCR assay. Vet. J., 195(3): 300-4.

14. Newton, J.R., Verheryen, K., Talbot, N.C., Timoney, J.F., Wood, J.L.N., Lakhani, K.H. and Chanter, N. (2000) Control of strangles outbreaks by isolation of guttural pouch carriers identified using PCR and culture of Streptococcus equi. Equine Vet. J., 32: 515-526.

15. Timoney, J.F. and Artiushin, S.C. (1997) Detection of Streptococcus equi in equine nasal swabs and washes by DNA amplification. Vet. Rec., 141: 446-447.

16. Alber, J., El-Sayed, A., Lammler, C., Hassan, A.A., Weiss, R. and Zschock, M. (2004) Multiplex polymerase chain reaction for identification and differentiation of Streptococcus equi subsp. zooepidemicus and Streptococcus equi subsp. equi. J. Vet. Med. B, 51: 455-458.

17. Baeur, A.W., Kirby, W.M.M., Sherris, J.C. and Truck, N. (1966) Antibiotic susceptibility testing by a standardized single disc method. Am. J. Clin. Pathol., 45: 493-496.

18. Timoney, J.F. (2004) The pathogenic equine streptococci. Vet. Res., 35: 397-409.

19. Sellon, D.C., Besser, T.E., Vivrette, S.L. and McConnico, R.S. (2001) Comparison of nucleic acid amplification, serology, and microbiologic culture for diagnosis of Rhodococcus equi pneumonia in foals. J. Clin. Microbiol., 39: 1289-1293.

20. Ijaz, M., Khan, M.S., Khan, M.A., Avais, M., Ali, M.M. and Saleem, M.H. (2011) Molecular identification and haematological values of strangles affected mules in Pakistan. Pak. J. Zool., 43(3): 587-592.

21. Malik, P. and Kalra, S.K. (2009) Prevalence of group C streptococci amongst equines in India. Indian J. Anim. Sci., 79: 459-465.

22. Ivens, P.A., Matthews, D., Webb, K., Newton, J.R., Steward, K., Waller, A.S., Robinson, C. and Slater, J.D. (2011) Characterization of 'strangles' outbreaks in the UK: The use of M protein typing of Streptococcus equi ssp. equi. Equine Vet J., 43: 359-364.

23. Bhardwaj, R.K. and Taku, A.K. (2010) An outbreak of strangles in horses. Indian Vet. J., 87: 810-811.

24. Dahlia, H., Maria, J., Jamaayah, A.Z., Suriani, M.N., Sharifah, S.H., Mahendran, R., Chandrasekaran, S. and Thang, S.L. (2004) Animal Health: A Breakpoint in Economic Development. The $11^{\text {th }}$ International Conference of the Association of Institutions for Tropical Veterinary Medicine and $16^{\text {th }}$ Veterinary Association, Malaysia, Congress. 23-24 August, 2004. p268-269.

25. Libardoni, F., Vielmo, A., Farias, L., Matter, L.B., Potter, L., Spilki, F.R. and deVargas, A.C. (2013) Diversity of seM in Streptococcus equi subsp. equi isolated from strangles outbreaks. Vet. Microbiol., 162(2-4): 663-9.

26. Jannatabadi, A.A., Mohammadi, G.R., Rad, M. and Maleki, M. (2008) Molecular identification of Streptococcus equi subsp. equi and Streptococcus equi subsp. zooepidemicus in nasal swabs samples from horses suffering respiratory infections in Iran. Pak. J. Biol. Sci., 11(3): 468-471.

27. Hansson, I., Johansson, K.E., Persson, M. and Riihimaki, M. (2012) The clinical significance of Nicoletella semolina in horses with respiratory disorders and a screening of the bacterial flora in the airways of horses. Vet. Microbiol., 162(2-4): 695-699.

28. Mir, I.A., Kumar, B., Taku, A., Faridi, F., Bhat, M.A., Baba, N.A. and Maqbool, T. (2013) Bacteriological and molecular detection of Streptococcus equi subsp. equi and Streptococcus equi subsp. zooepidemicus in equines of Northern India. J. Equine Sci., 24(3): 53-55.

29. Lindahl, S. (2013) Streptococcus equi subsp. equi and Streptococcus equi subsp. zooepidemicus Upper Respiratory Disease in Horses and Zoonotic Transmission to Humans Doctoral Thesis Submitted to Faculty of Veterinary Medicine and Animal Science Department of Clinical Sciences Uppsala. 
30. Timoney, J.F. and Mukhtar, M.M. (1993) The protective M proteins of the equine group C streptococci. Vet. Microbiol., 37: 389-395.

31. Prescott, J.F., Travers, M. and Yager-Johnson, J.A. (1984) Epidemiological survey of Corynebacterium equi infections on five Ontario horse farms. Can. J. Comp. Med., 48: 10-13.

32. Paillot, R., Darby, A.C., Robinson, C., Wright, N.L., Steward, K.F., Anderson, E., Webb, K., Holden, M.T., Efstratiou, A., Broughton, K., Jolley, K.A., Priestnall, S.L., Campi, M.C.M., Hughes, M.A., Radford, A., Erles, K. and
Waller, A.S. (2010) Identification of three novel superantigen-encoding genes in Streptococcus equi subsp. zooepidemicus, szeF, szeN, and szeP. Infect. Immun., 78(11): 4817-27.

33. Narwal, P.S., Monga, D.P. and Bhargava, D.N. (1994) Microbiological studies of reproductive disorder in equines. Indian J. Anim. Sci., 64: 51-55.

34. Uppal, P.K., Singh, B.K., Yadav, M.P. and Ghei, J.C. (1994) Identification and antibiogram of bacterial flora associated with reproductive disorders of mares. Int. J. Anim. Sci., 9: 57-58.

$* * * * * * * *$ 